\title{
Service Robot Management System for Business Improvement and Service Extension
}

\author{
Hideya Yoshiuchi*, Tomohiro Matsuda \\ Hitachi, Ltd., Research \& Development Group, Center for Technology Innovation - Digital Platform, 1-280, Higashi-koigakubo, \\ Tokyo,185-8601, Japan
}

\begin{tabular}{l} 
A R T I C L E I N F O \\
\hline Article history: \\
Received: 14 July, 2021 \\
Accepted: 26 October, 2021 \\
Online: 13 November, 2021 \\
\hline
\end{tabular}

Keywords:

Service Robot System

Data Analysis

External Equipment Association

\begin{abstract}
A B S T R A C T
Service robots are applied in many kinds of business fields and its various kinds of data can be collected with service robot in business scenes. Therefore, it becomes more important to utilize such data for business improvement. Additionally, due to limitation of design such as body size and battery life, a service robot cannot prepare much peripheral equipment on its body. In this paper, we will show two research results as for service robot. One is data analysis technology for business improvement as a function of operation and management system for service robot. Another is service extension of service robot by association of external equipment with proper design of utilization condition of external equipment. Through evaluation experiment, we confirmed potential effect of business improvement is $8.1 \%$ by modifying service scenario of robot.
\end{abstract}

\section{Introduction}

This paper is an extension of work originally presented in 5th International Conference on Robotics and Artificial Intelligence [1]. Due to the several social trends such as labor power and human resource issue, service robots technologies are being developed for realizing not only simple operation automation but also business improvement [1]. Recently, more people highly expect service robot system [2], [3] can solve social issues such as lack of labor power [4], improvement of productivity, human care [5] and so on. In manufacturing field, industrial robots were deployed and applied in 1970s for factory automation, and Internet of Things (IoT) technologies are utilized for productivity improvement and reduction of operation cost [6] with safe environment [7]. In service business field, service robots are expected to realize business and service quality improvement [8], [9] in the future aging society with fewer children. Based on these social and business background, we develop service robot and its application with intellectual media processing technology such as voice recognition and image analysis.

As for the robot deployment, people have higher attention to how to utilize various kinds of robots in actual fields for the purpose of collaboration with people. In industry field, Industry 4.0 [10] aims effective manufacturing with various technologies. Industrial robots are one of the most important technology in this fields. Industrial robots are designed to realize both production efficiency and safety for work with human in the same fields [11].

*Corresponding Author: Hideya Yoshiuchi, hideya.yoshiuchi.fq@hitachi.com

www.astesj.com

https://dx.doi.org/10.25046/aj060606
On the other hand, service robots are assumed to be applicable to many kinds of service and business scene through verbal and multimodal communication [12] not only for replacement of human labor load by automation but also for giving helpful function to people, therefore, applications for service robot have many variations for their purposes. For example, in a large facility such as an airport or a shopping mall, customers sometimes have difficulties for finding the way to their destination due to the scale and complexity of the facility. In such case, a service robot can give customers facility guidance information and support customers. In another case, a service robot can provide detail explanation of sold goods in a large shopping mall.

The value provided by service robots is considered in Businessto-Business-to-Consumer ( $\mathrm{B}$ to $\mathrm{B}$ to $\mathrm{C}$ ) scheme, and there are differences between the value of service provider (business side) expected and the value of consumer expected. Service providers apply service robots to their facilities for not only labor power but also some special skills such as multi-language guidance that normal employees cannot learn in short time. Consumers thinks service robots give them dedicated guidance. For example, when customers visit a large shopping mall for the first time and does not have enough knowledge for the mall, they ask a service robot where the shop that they are looking for. The service robot can give the location information by facility map, or the service robot can bring the customers to their destination by physical movement in the mall. 
People consider that service robots have much potential for social innovation. On the other hand, especially service providers, people would like to know how effective service robots are. Therefore, it is important to evaluate and visualize the effect of service robot for service providers and consumers. To evaluate service robots' effect by a kind of quantum key performance indicator (KPI), we have to clarify what service provider expect to service robots for their business improvement in each service field. After specifying KPI for business improvement measurement, we consider service scenario for robots and verify the effect of service robots operated by the service scenario with monitoring the specified KPI.

In addition to business improvement, it is important to consider how to realize applications by service robots with limited capability. Because service robot has several limitations such as body size and battery duration, it is difficult to prepare enough devices for applications to be realized by service robot. In such case, we need to consider how to extend capability of service robot for extended service.

In this paper, we explain two technologies. One is the service robot system and data analysis technology for business improvement. The other is external device association technology for service extension with service robot system. For both proposed technology, we will show evaluation results. The structure of this paper is as follows: In chapter 2, we will show basic architecture of service robot system. In chapter 3 , we will explain data analysis technology of service robot system for business improvement and its evaluation result. In chapter 4, We show external device association technology for service extension. Chapter 5 conclude this research.

\section{Service Robot System}

\subsection{Summary of Service Robot System Architecture}

Figure 1 shows general system architecture of service robot system. The service robot system consists of service robots and application servers that provides media processing function such as voice recognition, image processing and language processing. Additionally, the service robot system includes operation and management server that gives us management function for system operation such as machine condition monitoring and remote operation for emergency case. Because the main function of a service robot is service application through interaction with people, service robots are deployed in facilities of service providers. The methods of interaction between a service robot and people are sound and image. Therefore, a service robot has microphone and video camera for voice and image processing. A service robot system gathers voice and video data through microphone and video camera on a service robot, processes such media data, and provides basic interaction function to end users such as replying questions and giving useful information. Media processing function like voice recognition and video analysis consume much computational resources such as Central Processing Unit (CPU), sometimes media processing function is realized by application servers located out of a service robot.

Implementing media processing function by external application servers makes the size of a service robot smaller one. However, the data size of voice, image and video is relatively large and sending such media data from a service robot to application servers requires much larger network resources. Therefore, it is better to put application servers in the same service facilities as service robot's location. However, if it is not possible to put application servers in service facilities for some reasons such as facility size or no server room, application servers can be set in a management center.

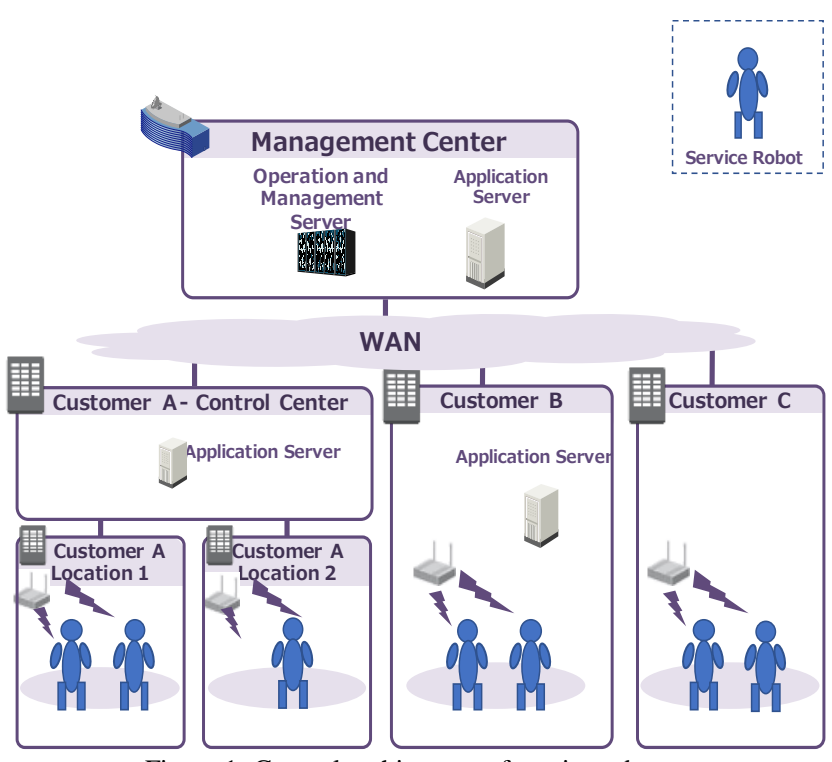

Figure 1: General architecture of service robot system

\subsection{Research Task of Service Robot System}

In service robot system, media processing is primitive function for interaction with end users. The combination of media processing realizes voice communication, and questions and answers. The one of the main purposes of developing service robot system is to organize service scenario by the set of communication and Q\&A list for various kinds of business scene such as guidance in shopping mall or large transportation facilities (ex. airports and railway stations). Service scenario consists of timing information of voice communication, contents of communication, gestures and movement of a service robot. Service scenarios are designed for satisfying business and service requirement of service provider. It is difficult to evaluate the effect of service robot system before service robot system start its operation. Therefore, one of the research challenges of service robot system is to establish the evaluation method of the application of service robot system for assumed KPI suitable for business and service requirement, and proposal scheme of service scenario modification to realize business improvement. Besides KPI issues, we have to consider how to realize service application with limited resource of service robot. If we set many devices on service robot, its size is getting bigger, and weight is getting heavier. Because service robots work among peoples, sometimes in busy crowdy area, it needs to keep human safety [13], [14]. If robots are bigger and heavier, service robots easily violate human safety. Therefore, robot developer has to consider not only functionality realized by various devices, but also risk assessment for safer robot operation [15], [16]. 


\section{Data Analysis Technology for Service Robot System}

In this section, data analysis technology for service robot system that realizes business improve is explained.

\subsection{System Architecture}

Figure 2 shows the architecture of service robot system with data analysis technology for business improvement. This system consists of the following components.

- Service robot: Communicate with end users by interaction with voice and video, gesture and movement and provides various kinds of service to the end users

- External equipment and system: Realize additional function that the service robot cannot provide such as GUI by digital signage.

- Application server: Providing media processing primitive function such as voice, video and language processing.

- Integrated service system: Store and manage all the data that can be collected in serlvice robot system. For the detail of collected later, we will explain in later section

- Business analysis server: Provide data analysis function for business improvement with all the data stored in the integrated service system.

- Customer business system: Various external system for customers' business purpose such as marketing, accounting and so on.

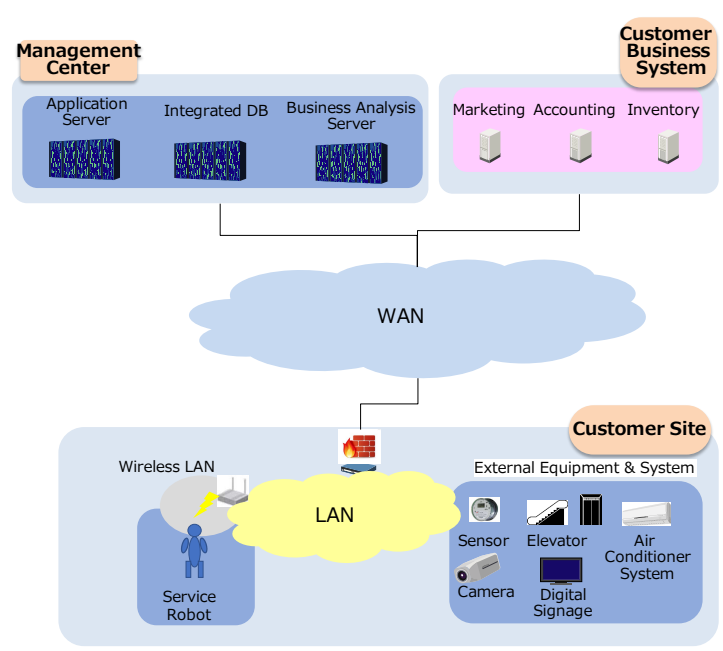

Figure 2 Architecture of Service Robot System with Data Analysis Technology

\subsection{Data Analysis for Business Improvement}

In service robot system, service robot provides various functions to end user by service scenario. The possible actions of a service robot consist of communication method by voice, image and language processing, Service scenario combines these actions. From the viewpoint of service provider, they expect service robots to do some business operations instead of their staff. The typical business operation realized by service scenario is guidance activity in commercial facilities or transportation facilities.

To realize business improvement, KPI related to business operation is measured and monitored. KPIs are indicators that evaluates business activities. For example, ISO 22400 defines important KPIs for manufacturing field mainly focusing on Manufacturing Execution System (MES) [17]. Commonly, KPIs focus on the following items:

- Cost: Cost KPI allows you to evaluate the activities that utilize economic resources in different processes. This can include energy consumption, cost of raw materials, cost of human resources and overhead costs.

- Time: Time KPI measures the time spent on an activity or a process. This can be the time from the beginning to the end of a process or a step of a process. Ways to measure time can be by the percentage of products or services delivered on time or the percentage of late deliveries.

- Productivity: Productivity KPI allows your organization to measure the efficiency of the operation. This can include measuring the percentage of downtime your equipment or machine experience. It also can be an output of operations such as product items or the number of served customers.

- Quality: Quality KPI help calculates the effectiveness of processes. These numbers may be based on the number of errors occurring and the number of completed processes. Other way to measure is the percentage of products that need corrective action and the number of satisfied customers.

Service robot applications have different tendency from industrial robot use case. Service robots are mainly used for support of end user's activities in service facilities such as commercial mall, railway station, airport, hospital and so on. Service robots mainly provide intellectual works to those customers; therefore, it is not always suitable to measure KPIs related to manufacturing productivity or automation activities. Suitable KPI candidates are service-related value such as service quality, the number of users that communicate with service robots and so on. After we decided to utilize service robot into our service environment, we have to choose KPIs that have relationship with service robot application. To analyze how the behavior of a service robot affects the KPIs, we need to monitor and store the data which describe service robots' activities. As for KPI evaluation, one or more service scenario affects the KPI value change. For example, daily sales information of commercial complex facilities is a sum of daily sales of each tenant. Some shop items are very popular and have strong effect to the daily sales of the tenant. In another viewpoint, daily sales information has relationship with likability of a facility.

To realize business improvement measured by KPIs, it is effective to figure out the service scenario that has strong relationship with business KPI and try to apply the service scenario as much as possible to service robots. For this purpose, we need to gather the enough data from service robot system for analysis of relationship between business KPI and service scenario. Then, we consider how to realize KPI improvement by modifying service scenario that much affects the KPI. The modification ways of the service scenarios are just candidate method and there is no assured result. Therefore, we must apply 
the modified service scenario to service robots and evaluate its efficiency by checking KPI increase or decrease. If the KPI is improved, we decide to apply scenario modification permanently. If the KPI decreases, we discard scenario modification and try another modification. The data type to be collected in service robot system for business improvement is as follows:

- $\quad$ KPIs to be improved as time-series data

- Service scenarios that are applied to service robots

- The type of service scenario

- Relationship between the KPI and service scenarios

- Execution result of service scenario

- Action history of service robots as time-series data

- Motion history of servicer robots: raising a hand, nod, showing bye-bye, etc.

- Image processing results

- Voicer recognition results

- Statements of service robots corresponding to the end users

Here we show the detail of data analysis process of service robot system for business improvement.

- Select one KPI for business improvement that a service provider applying service robot system would like to improve.

- Extract service scenario for service robot that has strong relationship with the KPI to be improved. The service scenario that gives very good effect to the KPI to be solved is selected the scenario that has possibilities for further business improvement.

- Analyze the data that relates to the extracted service scenario and decide whether the execution result of service scenario gives good or bad effect. By analyzing the result of service scenario execution and KPI change, we can understand tendency of service scenario and KPI behavior.

- Consider how to modify the extracted service scenario for business improvement: One of the ways to realize business improvement is to increase service scenario execution for good result.

- Modify the service scenario according to the way of step 4 and deploy the modified service scenario. After that, do evaluation test for a pre-determined term.

- $\quad$ Evaluating the KPI to be improved. If the KPI is improved, the service provider permanently applies the modification of the service scenario. If KPI does not change or show bad result, the service provider discards the modification and try to consider another modification way.

\subsection{Evaluation}

We applied service robot system to the exhibition. The task of a service robot is guidance of our company booth in the exhibition hall.

Figure 3 shows the layout of exhibition hall. The exhibition was held in 2 days and around 40,000 visitors came. In the exhibition, there were 171 topics are shown within 7 business categories. The service robot was set only in one category booth.
It has microphone, speaker and camera. By utilizing these devices, the service robot can communicate with people through verbal way. The service robot also has wheel and it can move around the exhibition hall, however, due to safety issue, we do not use moving function of the service robot. The contents of guidance are concierge service. The service robot waits for the visitors at the entrance of the booth and give the information related to the exhibition contents or facilities when visitors get close to the service robot. Additionally, stage show is periodically done and the service robot make announcement when the stage show begins soon. The basic behavior of the service robot for the exhibition is as follows:

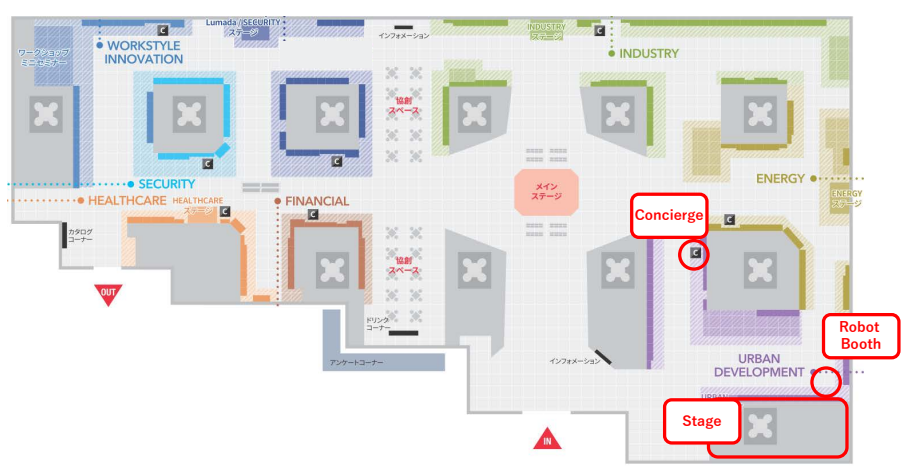

Figure 3: Architecture of Service Robot System with Data Analysis Technology

- The service robot waits for visitors' arrival at the entrance of the booth

- If there is no visitor, the service robot make announcement for its own service and try to get attention of visitors: "Hello, here we show our company's technology and service. Please come and visit our booth!”

- If the stage demo begins soon, the service robot makes announcement for the stage demo: "Our stage show starts soon. Please come and see the show!"

- When a visitor comes in front of the service robot, the service robot starts to talk and ask the visitor whether they have some questions or not.

- When the visitor asks a question, the service robot try to understand the question and reply answer. The assumed questions are questions for the exhibition contents, facility guidance and general question such as daily conversation.

- After answering a question, the service robot asks the visitor whether the visitor still have question or not.

- If the visitor has more question, the service robot continues to answer the question. If the visitor has no more question, the service robot finishes the service for the current visitor and wait for another visitor's arrival.

The service scerio of the service robot for the exhibition includes the following items:

- Announcement for the visitors

- Physical Motions that attract visitors' attention and promote natural communication

- Understanding the question by voice recognition and language processing

- Answering the question 
Service robot has two kind of tasks. The important task is guidance of exhibitions and it has higher priority than other task. When the service robot detects somebody's talking, it start guidance service to the person in front of the service robot. Service robots are recently deployed in actual fields. It has basic communication function through microphone and voice recognition technology. However, if people has no experience to communicate with service robot, they might have difficulty to let them understand what people say. The communication of service robots are controlled by computer program and it has detection function of human utterance section. Because not all the people understand this detection mechanism, sometimes communication gap happens between service robot and human. Semiotics[18] is an approach for smooth mutual communication. It sometimes applies sign or non-verbal communication method. In our system design, we applied sign of service robot to let the people understand the service robot is ready for communicating with the customers. For example, a service robot aim his face to the customer or raise its hand to start communication in natural way. The data related to the previous listed items are recorded as timeseries data and we used the data for business improvement analysis. For the business improvement analysis, there are two important tasks.

- Clarifying the KPI to be improved and related service scenario

- Making data formatting for data analysis of service scenario related data

Several KPI is suitable for evaluating the effect of exhibition. In this case, we focused on the understanding contents of exhibition by visitors. We set up one service robot in exhibition booth. It is difficult to measure how much the visitor understands exhibition contents, we chose the number of question and contents of question as a KPI that reflects visitors' interest to the exhibition. We classified the contents of question by judging how much the question relates to the exhibitions and monitored how the service robot behaves according to the service scenario. we recorded the data of service robot action for each visitor.

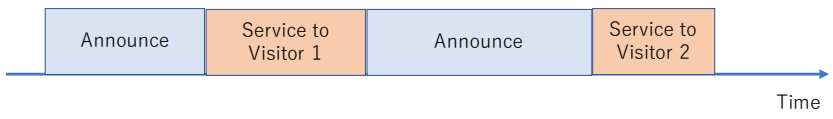

Figure 4: Structure of Concierge Service Data for Exhibition

Figure 4 shows the architecture of service robot action data for exhibitions. The data is classified into two categories. One is behavior with visitors, and the other is behavior without visitors. To separate time-series data of the service robot for each visitor, we used voice recognition result with specific words to indicate service start and end for a visitor. For example, when the service robot starts to ask question, the service robot says, "Are there any question?”. In the exhibition, the service robot serves to visitors for two hours and 25 minutes for 37 visitors. After we prepare data for each visitor, we extract the feature values that represents the data. In this evaluation, we chose the following items:

- The number of speaking of the service robot

- The number of voice recognition of the service robot (that means the number of speaking of a visitor)

- The number of motion that the service robot executes.

- The number of repeated questions

www.astesj.com

\section{- Service time for each visitor}

When the service robot cannot understand the meaning of the visitor's question, the service robot asks the visitor to repeat the same question once more. We calculated the feature values shown above, then we classified the result of service scenario for each visitor. We considered the best result of service scenario is directly related to the exhibition content because the purpose of service robot system for the exhibition is to let visitors understand the exhibition contents (that is, our company's technology and service). However, service robots are just now in the beginning stage and start to be deployed in actual world. Therefore, some visitors do not know what to ask to the service robot. In such case, visitors make the question for facilities such as "Where is a rest room?” or start daily life conversation. We inspected all the data for 37 visitors and classified the result of service scenario into the following four categories, then we give the label for the data for each visitor to represent the category.

- Answer the question for the exhibition contents

- Answer the question for facility information (such as restroom or exit)

- Start daily conversation

- Cannot understand the visitor's question and does not answer anything

Smaller number means visitors have higher interest for the exhibition contents. After data classification, we did cluster analysis with K-means method. We analyzed the clustering result with changing the number of clusters. When we set the number of clusters three, we confirmed the following tendencies.

- Cluster 1 (data of 22 visitors belongs to): In this class, the service time is relatively short, few visitors repeated their questions. The service robot answers a few questions.

- Cluster 2(data of three visitors belongs to): Service time is quite long. The visitor repeated question and asked so many questions, and the service robot answered all the related questions.

- $\quad$ Cluster 3 (data of 12 visitors belongs to): The service time is middle. In this class, visitors keep silent and the service robot try to make the visitor do some questions. However, visitors seem to have no idea and do not know what to be asked. Finally, the service robot regarded as the visitor has no question and ends the service for this visitor.

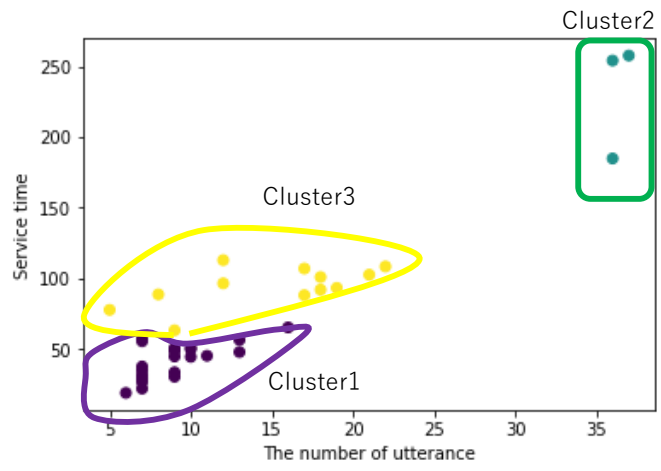

Figure 5: Clustering Result of Exhibition Data

Figure 5 shows the result of clustering. In Figure 4, service time and the number of utterance of the service robot are chosen as the 
evaluation axis that most reflects the clustering result. In cluster 1 , visitors and the service robot make smooth conversation. In some case, a visitor repeated the question, however, in most case, a visitor asked the exhibition contents and the service robot properly answered the question. In cluster 2, visitor seems to have some knowledge for service robots, and try to figure out what kind of questions. In this case, the service robot recognizes the visitors' question with high accuracy and the visitor and the service robot can talk long time. In cluster 3, the service robot cannot understand the questions, or the visitor does not know what to say.

We made detail analysis of the data classified into cluster 3 and figure out two major cases.

- Case1: The service robot failed to understand what the visitor says. The visitor quitted to communicate with the service robot.

- Case2: The visitor does not know what to ask to the service robot and there exists long silence time. Even though the service robot talks to the visitors and try to make the visitor ask some questions, the visitor has no idea and conversation is terminated.

As for the number of cluster, we have tested several cases. When we set the number of cluster as 4 , we confirmed the samples included in cluster 3 and cluster 4 show the similar features. Therefore, we chose 3 as the number of the cluster. In case 1, main reason is the accuracy of voice recognition and technology improvement is needed. In case 2, the issue is scenario design problem. In the evaluation experiment, the service robot just asked visitors whether the visitor has question or not. However, if we designed the service robot gives more detail information such as what kind of question is assumed, we can expect the service robots behaves to visitors more effective way. For case 2, three visitors are classified in this case. We defined service quality as the ratio of number of the visitors that the service robot properly gives service for exhibition demo. The number of whole visitor for service robot is 37 , that is, the sum of the data in all the clusters. If we can modify the service scenario and give better guidance to these three visitors, we can expect $8.1 \%$ (3/37) business improvement.

This is our first trial of service scenario analysis. Through this field test, we found the following issue to be solved in the future.

- In some cases, the service robot cannot correctly understand human speaking contents. In such case, we should provide alternative communication method by remote operation by human.

- Information on voice is sometimes not correctly understood by listener. In such case, visualizing method is useful for proper information providing. Since our service robot used in this field test does not have monitor, we should consider how to associate external device and the service robot in this application.

For further service scenario improvement, it is important to set target value of selected KPI, and making evaluation with Plan-DoCheck-Action cycle. Through continuous improvement, we can figure out root causes of the problem by utilizing analytic tools such as fishbone diagrams [19].

\section{Service Extension Technology with External Equipment} Association

In this chapter, we explain external device association technology for service robot system to realize service extension.

\subsection{Functions for External Equipment Association}

Figure 6 shows function blocks of external device association technology for the service robot system. Application server provides the following functions to realize external device association for service robots.

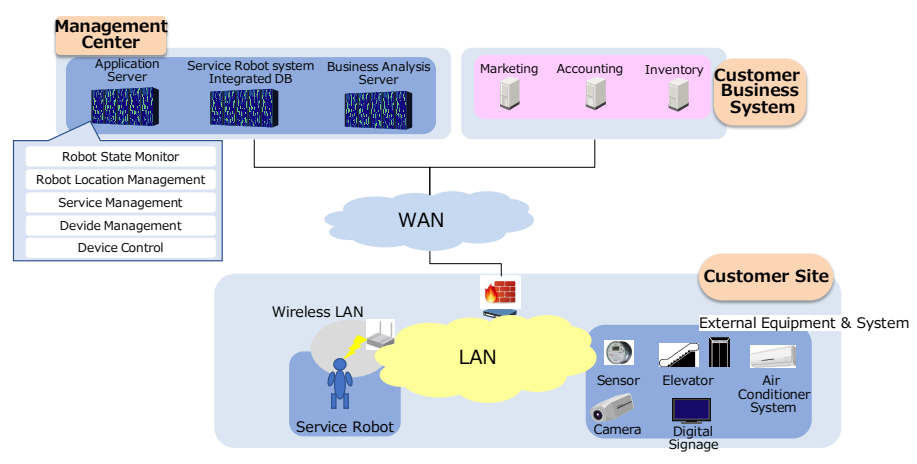

Figure 6: Functions for External Equipment Association

- $\quad$ Robot State Monitor: Collecting information on the status of various parts (motors, cameras, etc.) of service robot, then monitoring and visualizing the operating status of service robots. When some error occurs, this function notifies an alert.

- Robot Location Management: Managing location information of service robots based on the map information of a facility where service robots are working.

- Service Management: Managing services provided in facilities where service robots are installed. It includes the list of external equipment required for each service.

- Equipment Management: Managing equipment information about the installation location of external equipment in a facility and the conditions of use of external devices.

- Device Control: Provides an interface to control external equipment to realize various operation.

\subsection{Usage of External Equipment with Association Condition}

Service robot has several limitations on its design. Typical one is body size. If we set many peripheral equipment on a service robot, its size gets bigger and bigger. Even though we satisfy size limitation with great effort, many peripherals consume much electric power, and it requires larger battery. Therefore, service robot cannot prepare all the equipment for service realization and some equipment should be prepared as external devices. To solve this issue, we propose service management function that manages a list of devices required for services. The contents of service information are shown in Table 1.

For example, if a service robot does not have a display, an external display must be used to display visual information. The 
facility guidance service using a map is a typical example, and the facility guidance service can be provided only when the external display is available for a service robot.

Table 1: Service Information

\begin{tabular}{|l|l|}
\hline Item name & Description \\
\hline $\begin{array}{l}\text { Service } \\
\text { Identifier }\end{array}$ & Unique identifier of a service \\
\hline Device List & $\begin{array}{l}\text { Device list that is essential for a } \\
\text { service providing (ex. camera, } \\
\text { display, etc.) }\end{array}$ \\
\hline
\end{tabular}

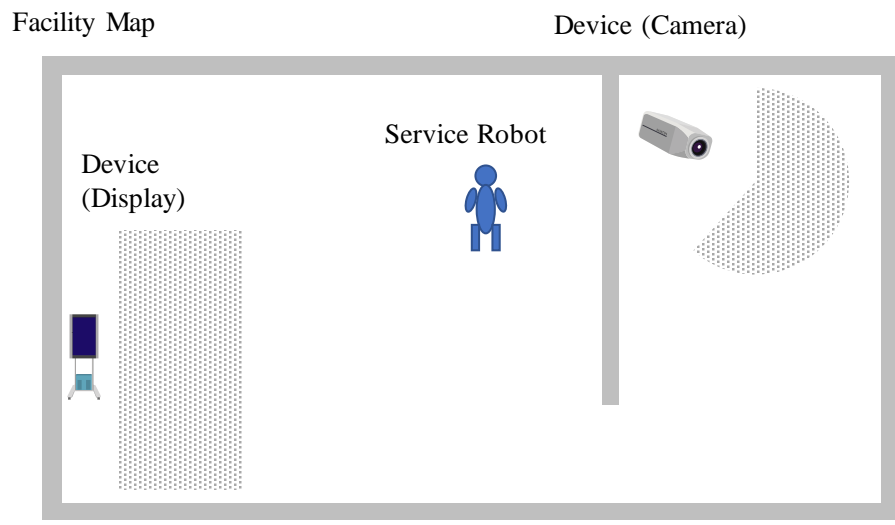

External device available zone

Figure 7: Example of external device availability

When a service robot starts to provide service, the service robot must check whether all the external devices necessary for the service are available or not in advance. The availability of external device depends on the position of external devices and a service robot. Also, we should consider whether the aimed devices are free and not used by other service robot. Figure 7 illustrates the positional relationship between a service robot and external devices. In this example, two external devices, a display and a surveillance camera, are installed in a facility. Taking a display as an example, for a service robot to use the display for facility information guidance service to end users such as facility users, the service robot must be close to the display. In addition, since the display has a display surface, the service robot needs to take care so that the display surface of the display can be seen by the end user. Based on this assumption, the available zone of the display is shown as the shaded area in Figure 7. The available zone of the camera differs depending on the service used, but if the recording area of the camera needs to include EMIEW, the available zone of the camera is the fan-shaped shaded area on the upper right of Figure 7. In either case, it is not enough to simply consider the physical distance between the service robot and the external devices.

Table 2 is a list of information managed by equipment management function. The external device has $\mathrm{X}$ and $\mathrm{Y}$ coordinates on the map created by using a laser range finder or the like as the position information of the device in the facility. In addition, information of available zone of external devices is stored as a list of parameters that express the range of area. Using these parameters, a service robot realizes extended service with external devices by the following procedure shown in Figure 8.

Table 2: Item List of Device Management

\begin{tabular}{|l|l|}
\hline Item name & Description \\
\hline Device Identifier & Unique identifier of a device \\
\hline Device Location & $\begin{array}{l}\text { Device location in a facility: X and } \\
\text { Y coordination in a map }\end{array}$ \\
\hline Device & $\begin{array}{l}\text { Device list that is essential for a } \\
\text { service providing (ex. camera, } \\
\text { Parameter List }\end{array}$ \\
\hline
\end{tabular}

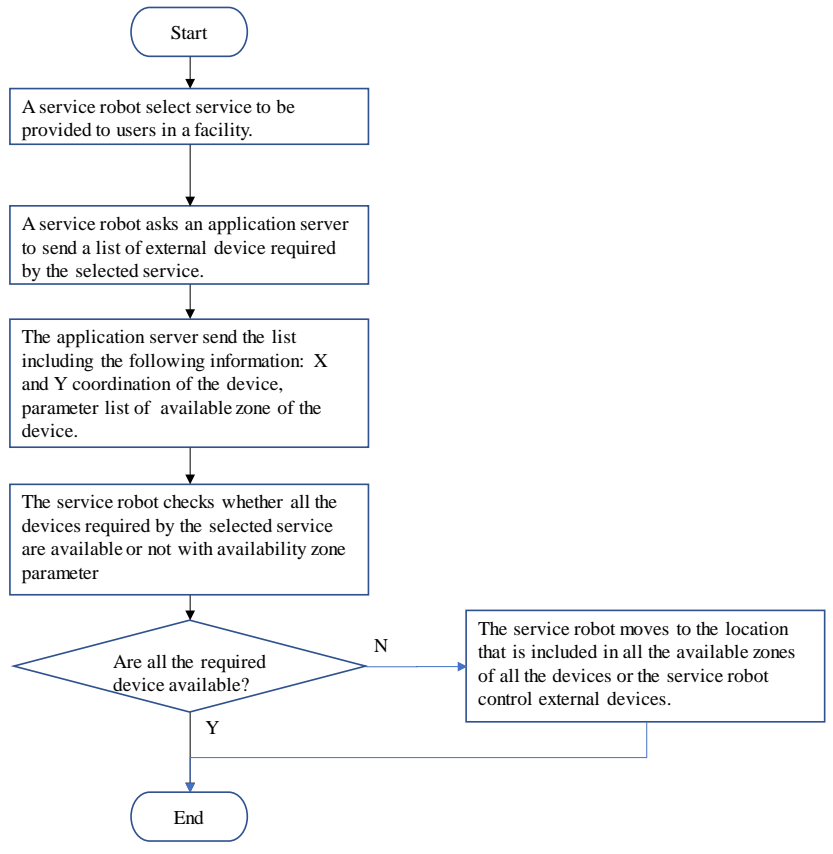

Figure 8: Flow chart of device availability check

\subsection{Evaluation}

In order to measure the effect of the proposed method, consider a concrete application example in a business field. As one of the typical application examples, let us consider a product and facility guidance service in a commercial facility. A typical commercial facility in Japan opens at $10 \mathrm{am}$ and closes at 9:00 pm. A service robot provides shoppers with on-site facility guidance and product explanation services for 11 hours when commercial facilities are open. Figure 9 shows the timetable for commercial facilities.

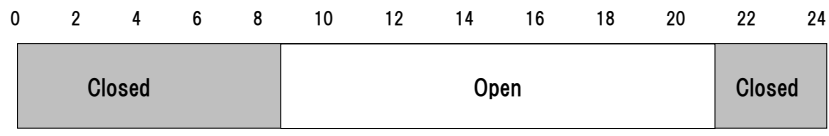

Figure 9: Timetable of the Shopping Mall 
Figure 10 shows the layout of the shopping mall. A shopping mall consists of an information center that guides visitors to the facility and a shopping area that sells products. A service robot shall provide facility guidance using digital signage at the information center and product explanations at the shopping area.

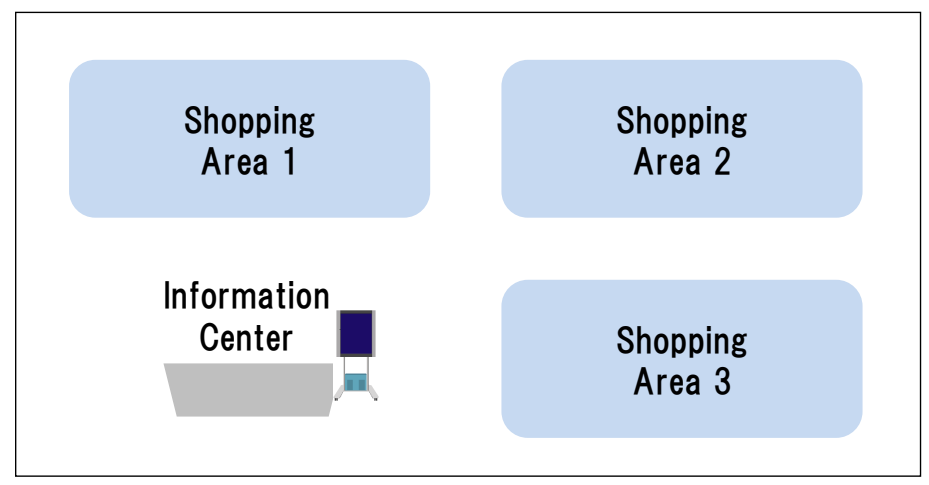

Figure 10: Layout of the shopping mall

Without the proposed method, the service robot cannot determine available services by comparing its location with the location of external devices. For this reason, shopping mall owner must set service robots at each location that provides different services. In this case, the arrangement of service robots is shown in Figure 11. In this case, 4 service robots are required, one in the information center and one in each shopping area.

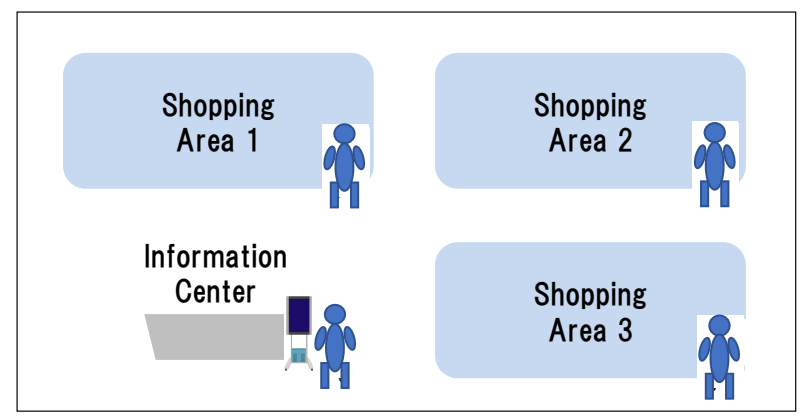

Figure 11: Arrangement of Service Robots with Conventional Technology

When applying the external device association technology, service robots can check whether its position is the information center and near the signage, or the location is the shopping center and away from the signage. Therefore, after providing facility information at the information center, it is possible to move to the shopping area and provide product information if necessary. We show the arrangement of service robots in this case in Figure 12. As for the association among service robots and signage, Simultaneous Localization And Mapping (SLAM) [20] technology is applied. The utilization area of signage is indicated in robot map managed by application server. The robot map has both signage utilization area and robot current location and application server can check whether signage is available by robot or not based on the robot location and signage condition. If robot is not at near signage, robot cannot use signage. Also, if the signage is used by another robot, signage is not available, too.

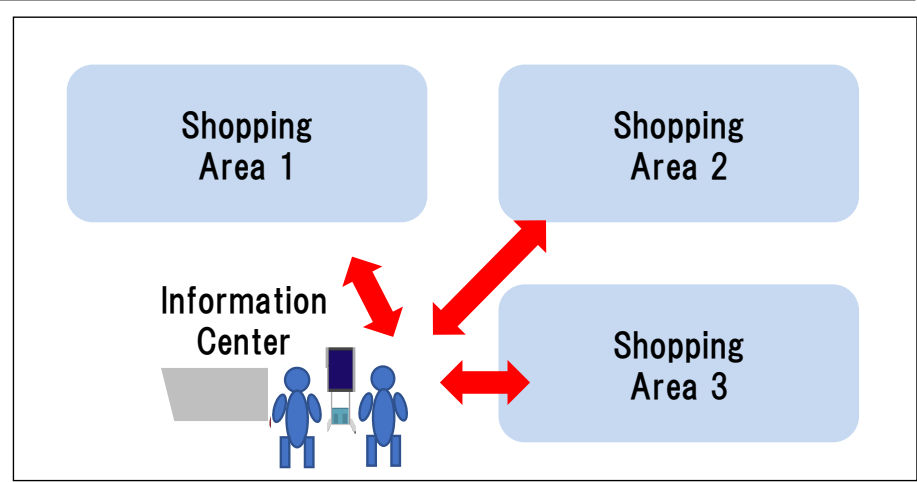

Figure 12: Arrangement of EMIEWs with Proposed Technology

The following conditions are assumed in the shopping mall.

- $\quad$ Time required for commercial facility guidance: 3 minutes

- Travel time between shopping area and information center: 1 minute

- $\quad$ Time required for product description: 5 minutes

- $\quad$ Visitor frequency: Once every 5 minutes

It takes 10 minutes for a service robot to move to the shopping area, explain the product, and return to the original position after guiding the commercial facility at the information center. If the customer's visit frequency is once every 5 minutes, it is possible to respond to customers without waiting for the customer with two service robots. In this case, the number of service robots installed in one commercial facility can be reduced by $50 \%$ compared to the conventional technology.

\section{Conclusions}

We developed data analysis technology of service robot system for business improvement and service extension with external device association with service robots. Through the evaluation by exhibition case, we found out the reason that the service robot failed to serve to the visitors, and how to modify the service scenario. $8.1 \%$ business improvement can be expected for exhibition case. In another case, we consider facility and product guidance service in a commercial facility and confirmed our proposed external device association technology can reduce the number of service robots set in a facility 50\% compared with conventional technology.

\section{Conflict of Interest}

The authors declare no conflict of interest.

\section{References}

[1] H.Yoshiuchi, T. Matsuda, and J. Dai, " Data Analysis Technology of Service Robot System for Business Improvement," Proceedings of the 2019 5th International Conference on Robotics and Artificial Intelligence (ICRAI), 7 11, 2019, doi:10.1145/3373724.3373733

[2] Z. Zhaohui, M. Xuesong, B. Xu, C. Hanghang and T. Jian, "Development of an intelligent interaction service robot using ROS," Proceedings of 2016 IEEE Advanced Information Management, Communicates, Electronic and Automation Control Conference, IMCEC 2016, 2017, doi:10.1109/IMCEC.2016.7867516

[3] C. Park, S. Kang, J. Kim and J. Oh, "A study on service robot system for elder care," 2012 9th International Conference on Ubiquitous Robots and 


Ambient Intelligence (URAI), IEEE, 2012,
doi;10.1109/URAI.2012.6463070

[4] T. Jyh-Hwa and L. Su Kuo, "The Development of the Restaurant Service Mobile Robot with a Laser Positioning System," Proceedings of the 27th Chinese Control Conference, IEEE, 2008, doi:10.1109/CHICC.2008.4605839

[5] H. S. Ahn, J. Choi, H. Moon, M. Jang, S. S. Kwak and Y. Lim, "Social Human-Robot Interaction of Human-Care Service Robots," 2019 14th ACM/IEEE International Conference on Human-Robot Interaction (HRI), 2019, doi:10.1109/HRI.2019.8673282

[6] D. J. Ahn, J. Jeong and S. Lee, "A Novel Cloud-based Fog Computing Network Architecture for Smart Factory Big data Applications," 2018 SouthEastern European Design Automation, Computer Engineering, Computer Networks and Society Media Conference (SEEDA_CECNSM), 2018, doi:10.23919/SEEDA-CECNSM.2018.8544946

[7] M. Dohi, K. Okada, I. Maeda, S. Fujitani and T. Fujita, "Proposal of Collaboration Safety in a Coexistence Environment of Human and Robots," 2018 IEEE International Conference on Robotics and Automation (ICRA), 2018, doi:10.1109/ICRA.2018.8460869

[8] T. Savickas and O. Vasilecas, "Business Process Event Log Use for Activity Sequence Analysis," 2015 Open Conference of Electrical, Electronic and Information Sciences (eStream), 2015, doi:10.1109/eStream.2015.7119500

[9] G. Park, L. Chung, L. Khan and S. Park, "A Modeling Framework for Business Process Reengineering Using Big Data Analytics and a Goalorientation," 2017 11th International Conference on Research Challenges in Information Science (RCIS), 2017, doi: 10.1109/RCIS.2017.7956514

[10] K. Zhou, T. Liu and L. Zhou, "Industry 4.0: Towards future industrial opportunities and challenges," 2015 12th International Conference on Fuzzy Systems and Knowledge Discovery (FSKD), 2147-2152, 2015, doi: 10.1109/FSKD.2015.7382284.

[11] J. A. Marvel, J. Falco and I. Marstio, "Characterizing Task-Based HumanRobot Collaboration Safety in Manufacturing," IEEE Transactions on Systems, Man, and Cybernetics: Systems, 45(2), 260-275, 2015, doi: 10.1109/TSMC.2014.2337275.

[12] Y. Kim and W. C. Yoon, "Generating Task-Oriented Interactions of Service Robots," IEEE Transactions on Systems, Man, and Cybernetics: Systems, 44(8) , 2014, doi: 10.1109/TSMC.2014.2298214

[13] G. Yu, S. Hong, K. Sung and J. Seo, "A study on the risk investigation and safety of personal care robots," 2017 17th International Conference on Control, Automation and Systems (ICCAS), 904-908, 2017, doi:10.23919/ICCAS.2017.8204353

[14] A. J. Kim, B. B. Kang, C. J. Shin, D. H. Kim and E. S. Jang, "A Study of Safety Requirements of Service Robot Appliances for Household and Similar Use," 2019 19th International Conference on Control, Automation and Systems (ICCAS), 1410-1411, 2019, doi:10.23919/ICCAS47443.2019.8971666

[15] W. Wang, Z. Wang, F. Gao and Y. Ma, "Design of Wireless Remote Control Service Robot," 2020 IEEE International Conference on Information Technology,Big Data and Artificial Intelligence (ICIBA), 651-654, 2020, doi: 10.1109/ICIBA50161.2020.9276882

[16] S. B. Lee and S. Hun Yoo, "Design of the companion robot interaction for supporting major tasks of the elderly," 2017 14th International Conference on Ubiquitous Robots and Ambient Intelligence (URAI), 655-659, 2017,doi: 10.1109/URAI.2017.7992695

[17] K. Kumagai, M. Fujishima, H. Yoneda, S. Chino, S. Ueda, A. Ito, T. Ono, H. Yoshida and H. Machida, "KPI element information model (KEI Model) for ISO22400 using OPC UA, FDT, PLCopen and AutomationML," 2017 56th Annual Conference of the Society of Instrument and Control Engineers of Japan (SICE), 602-604, 2017, doi: 10.23919/SICE.2017.8105550.

[18] W. Takano and Y. Nakamura, "Statistically integrated semiotics that enables mutual inference between linguistic and behavioral symbols for humanoid robots," 2009 IEEE International Conference on Robotics and Automation, 646-652, 2009, doi: 10.1109/ROBOT.2009.5152574.

[19] O. A. Makinde, T. Munyai and B. I. Ramatsetse, "Establishing suitable process improvement methodologies for optimizing servicing operations in the banking industries," 2017 IEEE International Conference on Industrial Engineering and Engineering Management (IEEM), 860-864, 2017, doi: 10.1109/IEEM.2017.8290014.

[20] F. Hashikawa and K. Morioka, "Mobile robot navigation based on interactive SLAM with an intelligent space," 2011 8th International Conference on Ubiquitous Robots and Ambient Intelligence (URAI), 788-789, 2011, doi: 10.1109/URAI.2011.6146017. 